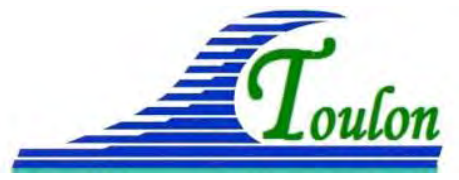

XIV èmes Journées Nationales Génie Côtier - Génie Civil Toulon, 29 juin au $1^{\text {er }}$ juillet 2016

DOI:10.5150/jngcgc.2016.020 (C) Editions Paralia CFL disponible en ligne - http://www.paralia.fr - available online

\title{
Travaux de protection et de mise en valeur du lido de Frontignan
}

\author{
Marie COUTOS ${ }^{1}$, François HACQUES ${ }^{1}$
}

\section{ARTELIA EAU \& ENVIRONNEMENT, 6 rue de Lorraine, 38130 Echirolles,} France.

Marie.coutos-thevenot@arteliagroup.com ; Francois.hacques@arteliagroup.com

\section{Résumé :}

L'étude présentée ici porte sur les travaux de protection du Lido de Frontignan, et plus particulièrement des secteurs de la Dent Creuse et des Aresquiers qui s’étendent sur un linéaire cumulé d'environ $2 \mathrm{~km}$.

Il est présenté :

- la dynamique hydro-sédimentaire du site à laquelle le littoral est soumis,

- les risques de rupture du cordon littoral,

- les solutions de protection qui ont été proposées et mises en place : reconditionnement des épis existants, construction de nouveaux épis, rechargement de l'estran, mise en place d'accès à la plage,

- le déroulement des travaux réalisés en 2015, avec un focus sur le rechargement,

- les mesures de réduction des impacts qui ont été employées au cours de ces travaux.

Mots-clés : Génie côtier, Hydro-sédimentaire, Protection côtière, Travaux maritimes, Dragage, Environnement littoral.

\section{Introduction}

Le lido de Frontignan est situé dans le département de l’Hérault (34), sur la commune de Frontignan - La Peyrade. Ce lido sépare la mer d'un système lagunaire important (étang des Mouettes, une partie de l'étang d'Ingril et la partie ouest de l'étang de Vic).

Le lido s'étend du port de pêche et conchylicole à l'Ouest jusqu'au littoral des Aresquiers à l'est (au droit du pont des Aresquiers). Il peut être caractérisé comme suit :

a) la zone urbanisée de Frontignan qui s'étend sur 5,4 km de part et d'autre du port de plaisance soit depuis le port de pêche jusqu'au camping des Tamaris inclus. Ce secteur est équipé de nombreux ouvrages maritimes (épis, épis en T, épis en L).

b) la zone de la Dent Creuse située à l'est du camping des Tamaris. Longue d'environ 0,5 km, elle présente des aménagements terrestres plus diffus (route, parkings, zone de lagunage...) et ne comporte pas d'ouvrage de protection.

c) enfin, le littoral des Aresquiers, constitué par :

- au Sud-Ouest, un littoral protégé par 6 épis.

- au Nord-Est : la plage des Aresquiers, longue d'environ 1,3 km, constituée d'une plage sèche en sable très étroite, de quelques mètres de large, appuyée contre un 


\section{Thème 2 - Dynamique sédimentaire}

cordon de galets présent sur le haut de plage. Ce cordon est entretenu par la commune de Frontignan. Hormis ce cordon et les vestiges d'un ancien épi à peine émergeant, il n’y a pas d'autres ouvrages de défense contre la mer sur ce tronçon du littoral.

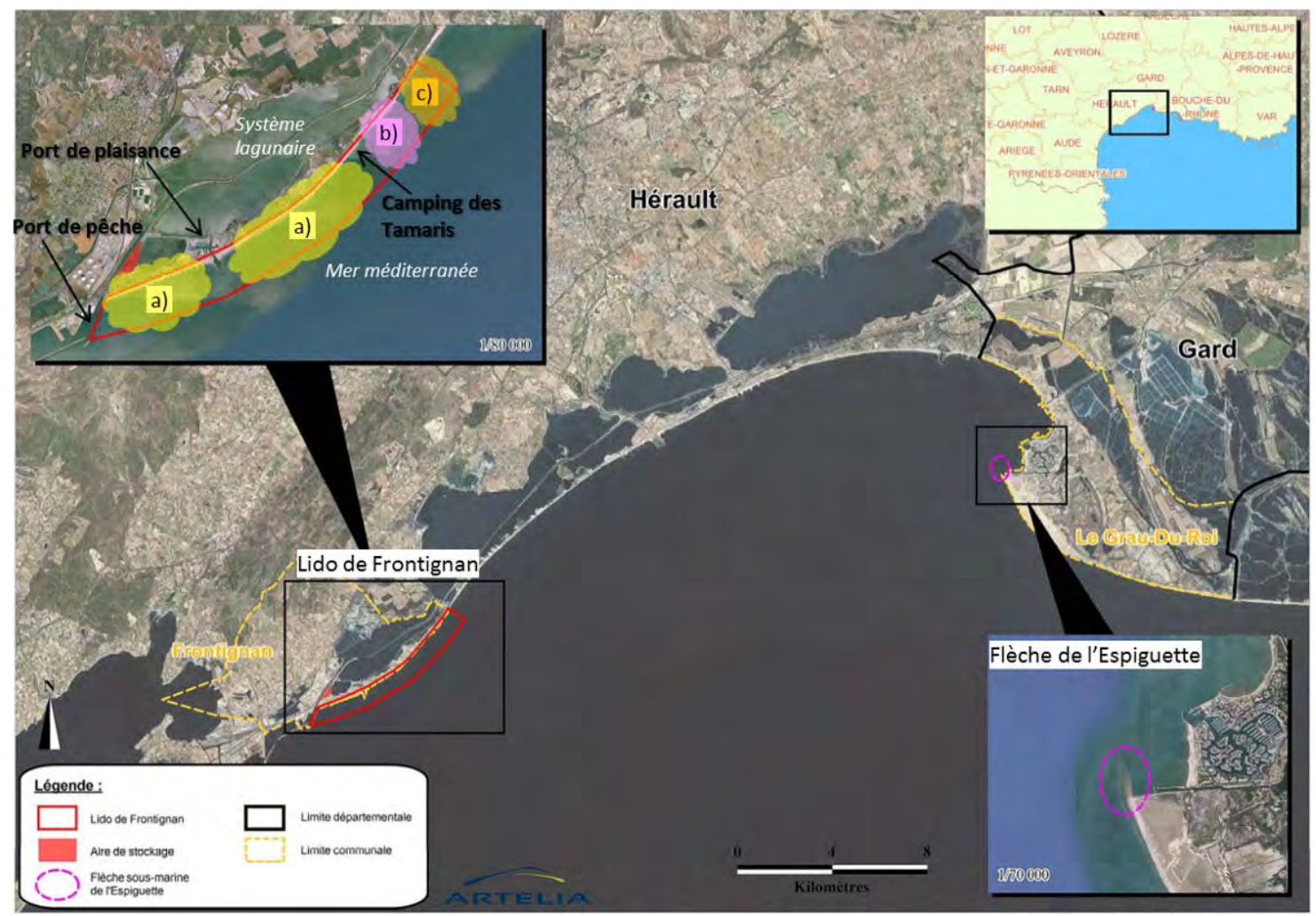

Figure 1. Localisation de la zone d'étude.

La première tranche de travaux réalisée en 2015 concernait deux secteurs, l'un en extrémité Ouest (port de pêche) sur environ 500 m, l'autre côté Est (Dent Creuse et Aresquiers) sur environ $1700 \mathrm{~m}$. Cet article présente les aménagements qui ont été réalisés sur ce dernier secteur, comprenant la Dent Creuse et les Aresquiers.

\section{Présentation du risque maritime pour les secteurs de la Dent Creuse et des Aresquiers}

Le littoral est exposé aux agitations provenant du secteur Est à Sud-Ouest et a subi plusieurs tempêtes majeures au cours des 30 dernières années avec des hauteurs significatives de houle fortes (Hs de 5,5 à $7 \mathrm{~m}$ mesurées par les bouées du réseau CANDHIS, site web, du CETMEF). Les niveaux de mer exceptionnels pendant ces évènements de tempête atteignent de l'ordre de +1 m IGN69 compte tenu de la surélévation du niveau moyen du fait des facteurs météorologiques et hydrodynamiques. 


\section{XIV ${ }^{\text {èmes }}$ Journées Nationales Génie Côtier - Génie Civil \\ Toulon, 29 juin au $1^{\text {er }}$ juillet 2016}

Le littoral de la Dent Creuse et des Aresquiers est particulièrement vulnérable face à de tels évènements hydrauliques exceptionnels du fait :

a) de la faible altitude de l'estran et de la zone arrière littoral comprise entre +1 et $+2 \mathrm{~m}$

IGN69 (l'aléa de référence pour la submersion marine étant estimée à +2 m IGN69),

b) de la faible largeur du cordon dans ce secteur.

Du fait des franchissements, le cordon se trouve généralement fortement dégradé après la période hivernale et il est régulièrement restauré par la commune. Cette protection permet de limiter les franchissements, mais reste toutefois insuffisante pour protéger l'arrière plage lors de tempêtes exceptionnelles.

\section{Les conditions hydro-sédimentaires}

Pour définir les conditions hydro-sédimentaires du littoral, il a été estimé :

a) les intensités et directions des transits littoraux empiriquement (voir Shore Protection Manual, CERC, 1984), conduisant à un transit littoral annuel résultant de 30000 $\mathrm{m}^{3}$ /an vers le Nord-Est.

b) les évolutions passées du littoral par comparaison des photographies aériennes de 1986 à 2009 et des levés topo-bathymétriques de 2002, avril 2007 et mars 2009. Cette analyse a mis en évidence:

- la stabilité des plages dans la zone protégée par des épis,

- partout ailleurs, une tendance érosive du littoral avec un taux annuel moyen compris entre $-1,0$ à -2,0 m/an sur les 23 dernières années.

c) le risque de submersion marine, modélisé par la mise en œuvre du logiciel Xbeach, pour la condition hydraulique de référence (condition décennale du type de la tempête de 1982 ou 2003).

Le graphique ci-dessous présente les caractéristiques de la partie aérienne du profil type (cotes supérieures à 0m IGN) testées en faisant apparaître l'emplacement de la route et du cordon d'arrière plage. On peut s'apercevoir qu'en 2009 le lido ne faisait plus qu'une quarantaine de mètres de large.

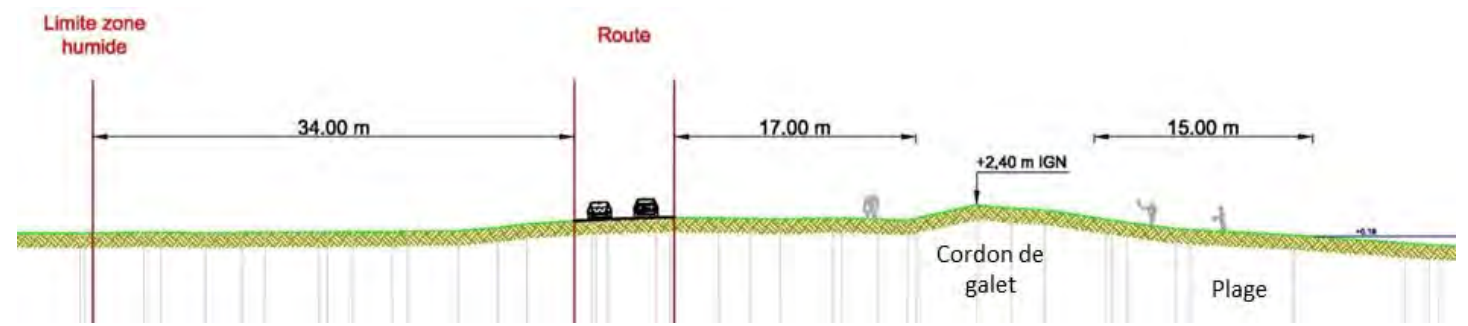

Figure 2. Représentation du profil type au-dessus de 0 m IGN (levé LIDAR 2009).

Les résultats (voir figure suivante) montrent que le lido des Aresquiers est soumis à un risque majeur de destruction du cordon en l'espace des quelques heures d'une tempête. 


\section{Thème 2 - Dynamique sédimentaire}

Le phénomène d'érosion du cordon lors des tempêtes ne fait que renforcer cette fragilité au cours du temps. Les évolutions passées montrent qu'en l'absence de toute intervention sur le littoral, une brèche pourrait s'ouvrir entre la mer et l'étang d'Ingril, permettant à la mer de pénétrer facilement à l'intérieur des terres (horizon > 20 ans).

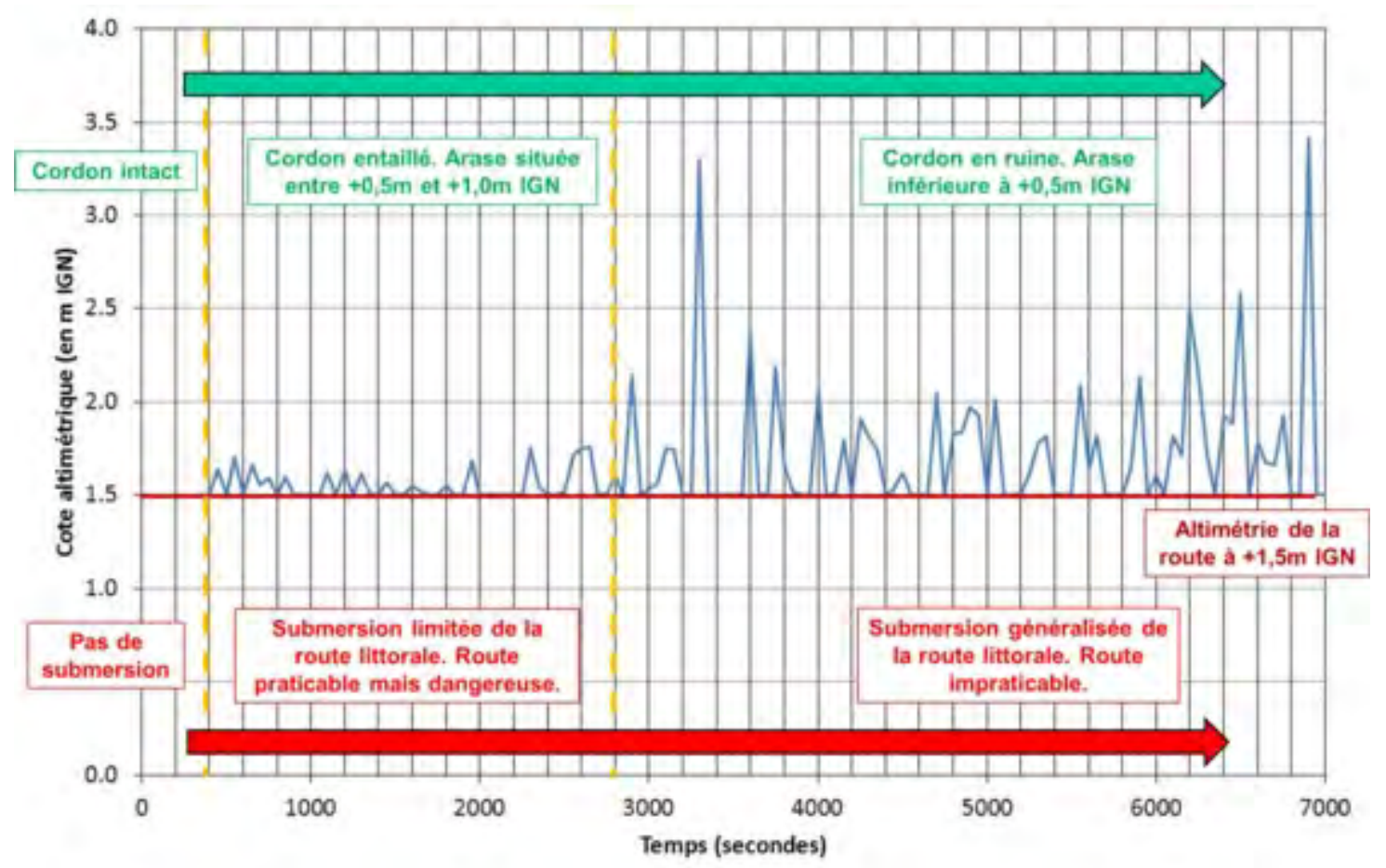

Figure 3. Evolution du niveau d'eau sur la route littorale en fonction de l'état du cordon d'arrière-plage durant une tempête de référence

$(\mathrm{Hm} 0=6 m-T p=10$ s - Niveau d'eau $=+1,19 m I G N)$.

\section{Descriptif des travaux de protection du littoral de la Dent Creuse et des Aresquiers}

Face à ces analyses, les travaux de protection du lido de Frontignan au niveau des secteurs de la Dent Creuse et des Aresquiers ont consisté à :

a) Reconditionner les épis existants de manière à obtenir des épis de longueur dégressive pour favoriser la transition sédimentaire entre la zone protégée et la zone naturelle.

b) Construire 3 nouveaux épis de longueur dégressive.

c) Renforcer le cordon dunaire en galets pour préserver la route littorale des intrusions marines.

d) Réalimenter les plages et petits fonds en sable d'un volume de $200500 \mathrm{~m}^{3}$ et favoriser le déferlement anticipé des vagues.

e) Aménager des accès à la plage (passerelles en bois) au niveau du cordon. 


\section{XIV ${ }^{\text {èmes }}$ Journées Nationales Génie Côtier - Génie Civil \\ Toulon, 29 juin au $1^{\text {er }}$ juillet 2016}

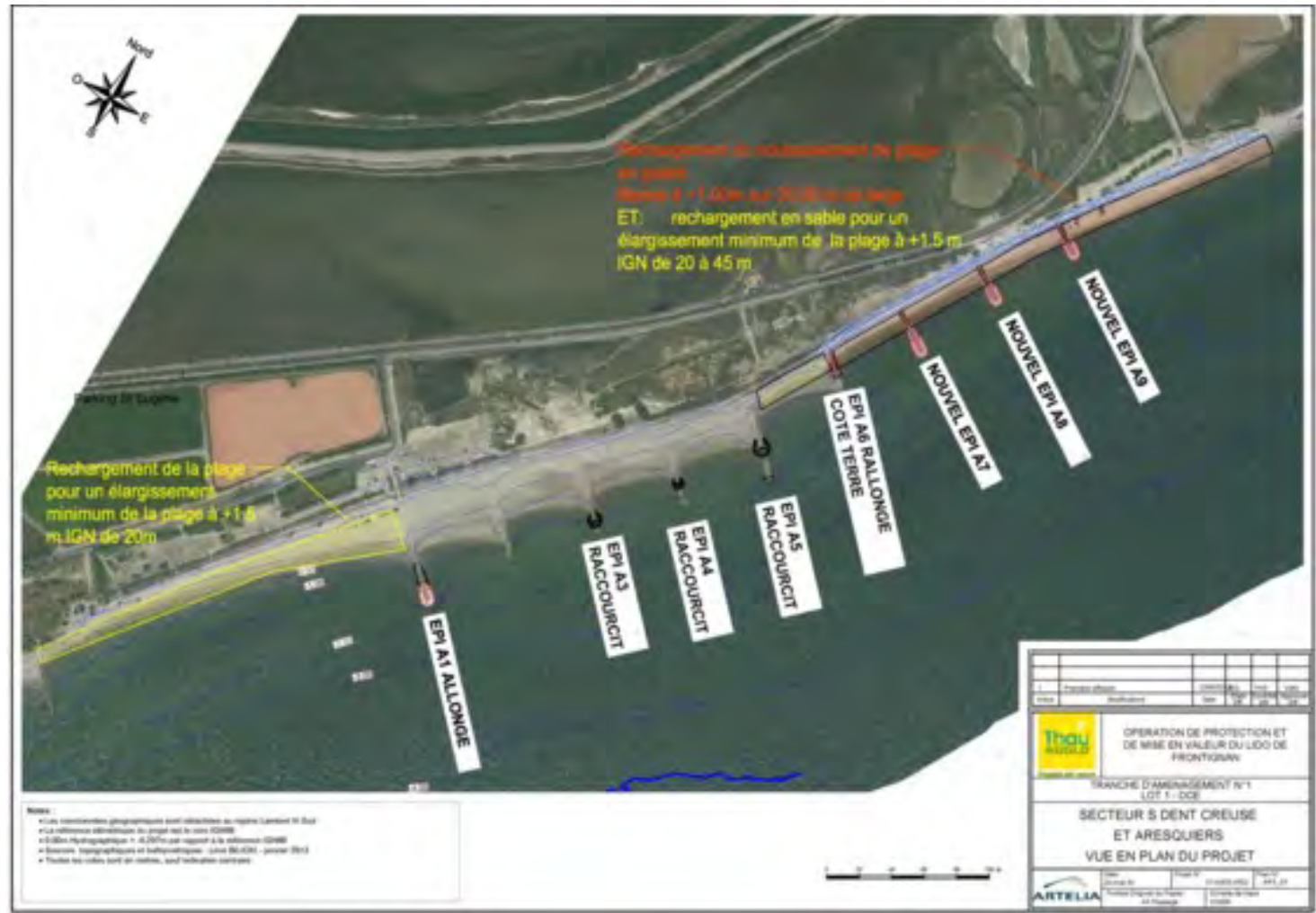

Figure 4. Aménagements réalisés pour les secteurs Dent Creuse et des Aresquiers.

\section{Déroulement des travaux : du sable aux passerelles en passant par les galets}

Le principe de la protection du cordon des Aresquiers est le suivant :

- création d'un cordon en galets à +3,5m IGN,

- mise en place d'un soubassement de galets en pied du cordon pour limiter les abaissements des fonds en tempête,

- couverture de surface en galets sur la zone de plage sèche,

- apports de sable,

- construction des ouvrages de franchissements du cordon (escaliers bois).

La coupe type du profil de rechargement est donnée sur la figure 5 pour le secteur de des Aresquiers.

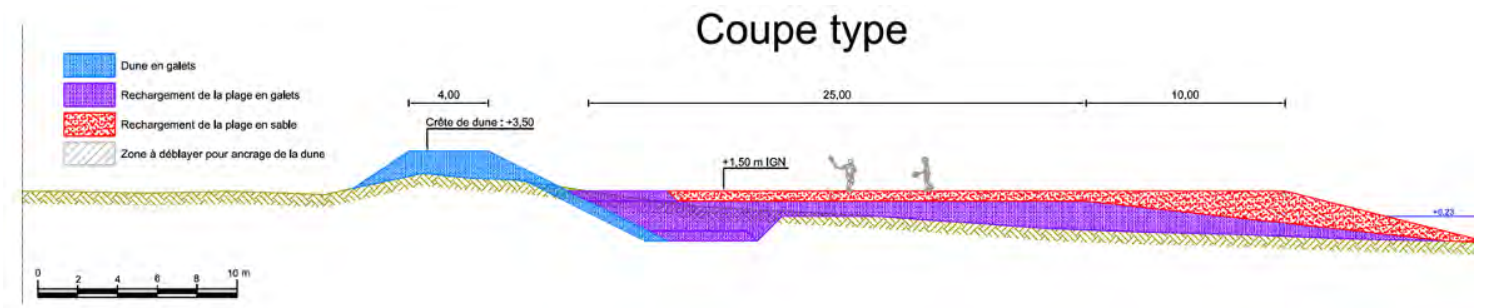

Figure 5. Coupe type du profil de rechargement aux Aresquiers. 


\section{Thème 2 - Dynamique sédimentaire}

Les travaux de rechargement en sable ont été réalisés en 1 mois à la fin de l'hiver 2015 selon le schéma suivant :

a) dragage de la flèche sédimentaire sous-marine de l'Espiguette à l'aide d'une drague aspiratrice en marche (drague THOR) et navigation de la drague jusqu'au lieu de rechargement.

b) refoulement de la mixture et rechargement.

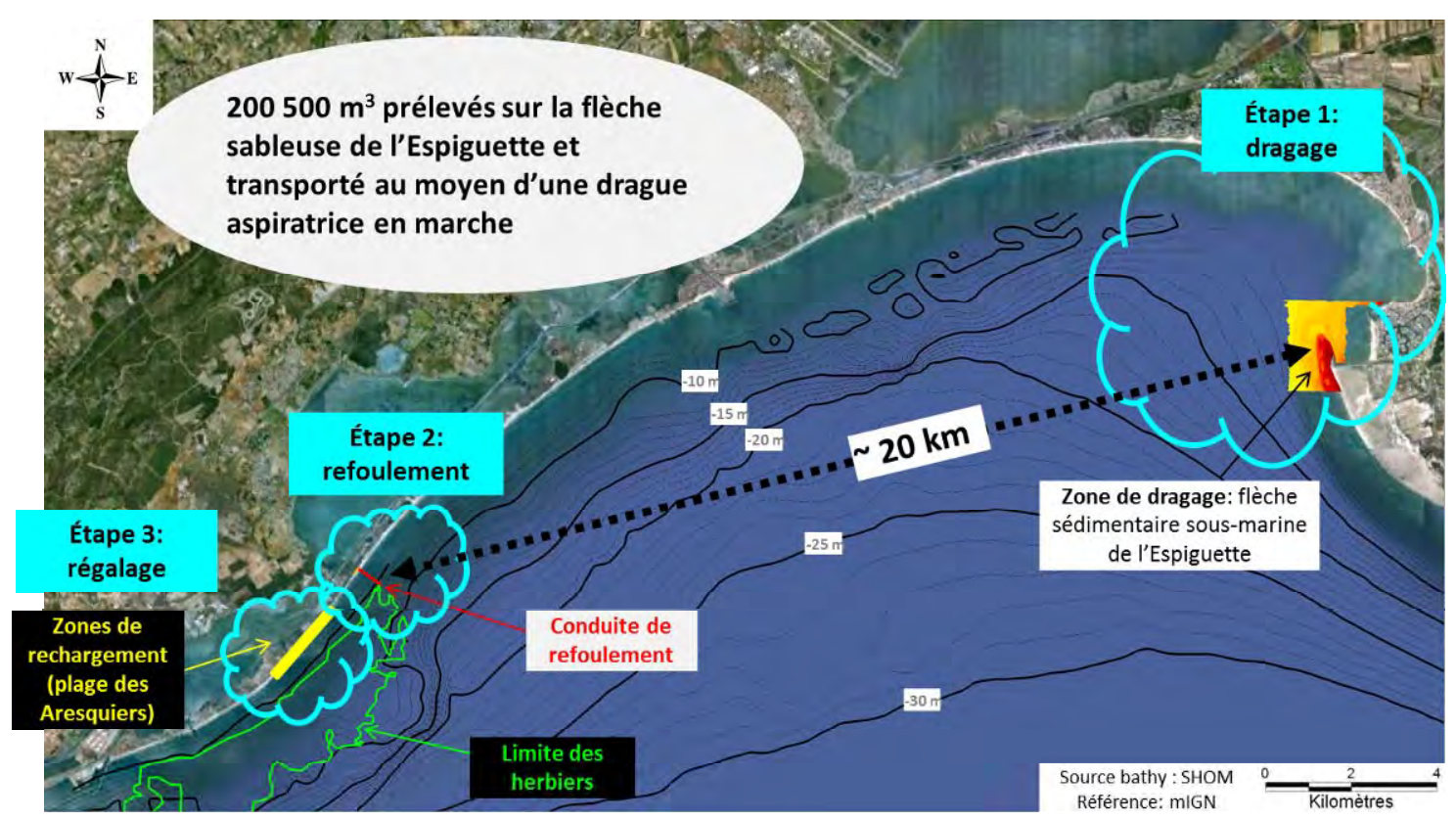

Figure 6. Schéma du cycle de dragage et de l'opération de rechargement de la plage.

Compte-tenu de la présence d'herbiers de posidonies au droit du site à recharger, le refoulement sur la plage s'est effectué selon un protocole spécifique de manière à limiter au maximum l'augmentation de la turbidité pendant les travaux.

La méthode suivante a donc été employée :

- connexion de la drague à une bouée littorale située hors des herbiers de posidonies et à environ $500 \mathrm{~m}$ de la plage, par -6 m IGN de profondeur ;

- refoulement de la mixture dans des casiers de décantation (bassins de ressuyage) creusés sur la plage de manière à faciliter la décantation des sables à partir de la mixture de dragage.

- reprise les sables refoulés et décantés par des pelles mécaniques, chargement sur des tombereaux, et transport du sable sur chaque secteur à recharger.

- les eaux de ressuyage étaient canalisées au Nord-Est du site (hors herbiers de posidonies) via une conduite placée sur la plage puis rejetée en mer. 


\section{XIV ${ }^{\text {èmes }}$ Journées Nationales Génie Côtier - Génie Civil \\ Toulon, 29 juin au $1^{\text {er }}$ juillet 2016}

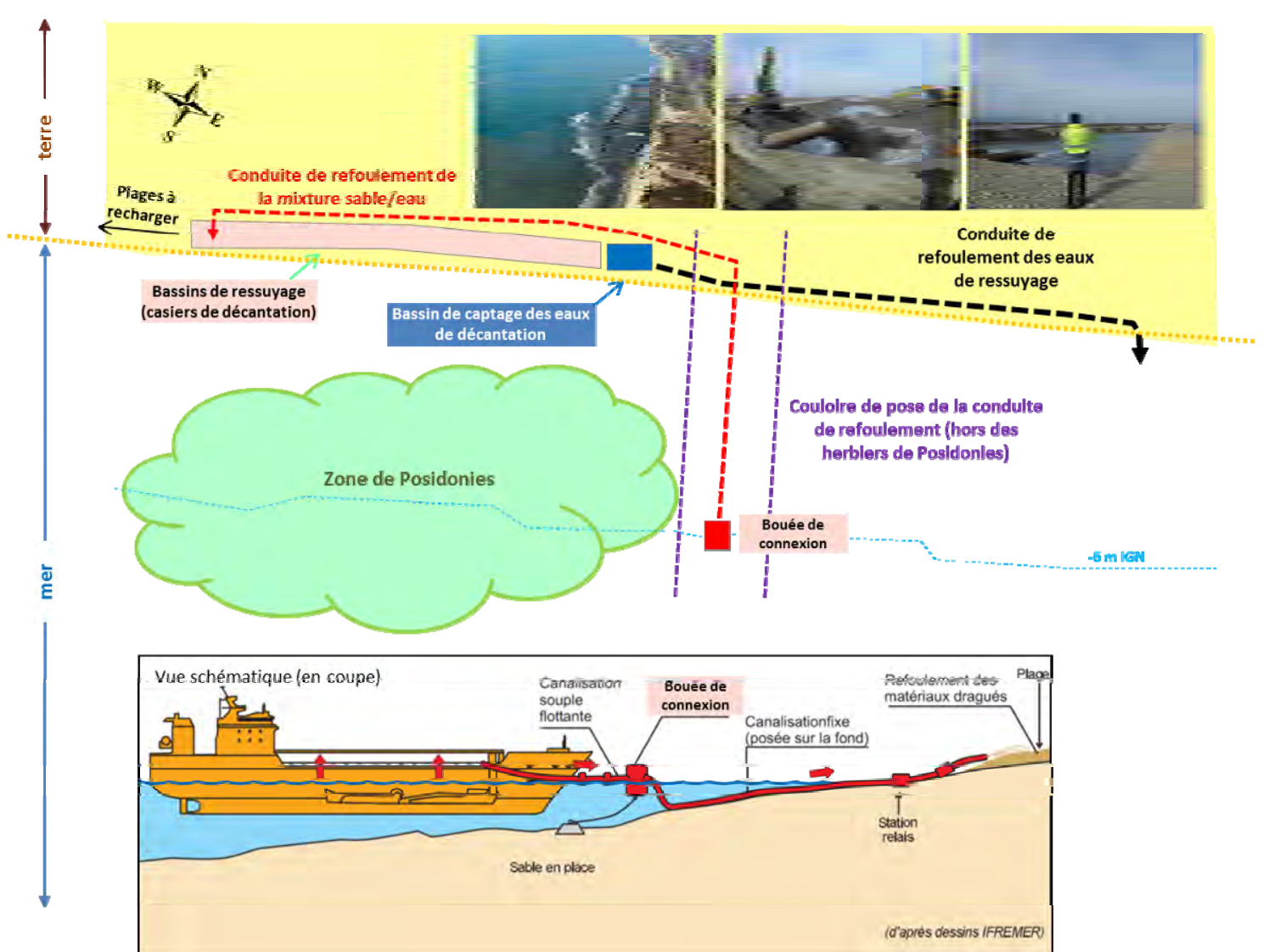

Figure 7. Schéma explicatif de la procédure de refoulement de la mixture draguée.

Le secteur des Aresquiers se situe dans une zone soumise à différentes réglementations (zones ZNIEFFs, Natura 2000, présence de 4 habitats naturels d'intérêt majeur et communautaire, 5 espèces à forte valeur patrimoniale, herbier de posidonies, ...).

De nombreuses mesures de réduction des impacts ont été mises en place lors des travaux avec :

- le balisage des voies de circulation sur le site afin de protéger les zones sensibles,

- l'absence de travaux sur les zones de nidification durant les mois d'avril à mi-août,

- la protection des herbiers de posidonies : suivi de la turbidité, survols aériens à toutes les étapes du chantier,

- la conservation du stock de graine et des stations d'Euphorbe péplis, mise en culture par un pépiniériste et réensemencement in situ à la fin des travaux,

Les photos de la figure 9 présentent la plage une fois les travaux terminés. 


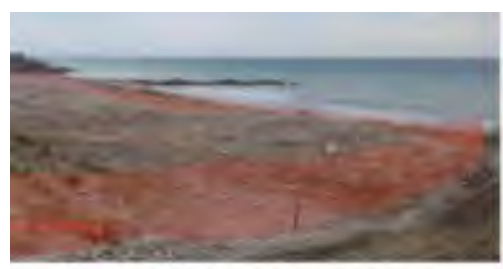

Balisoge Euphorbe Pelplis (source: Asconit, prospections himernales, janvier 2015)

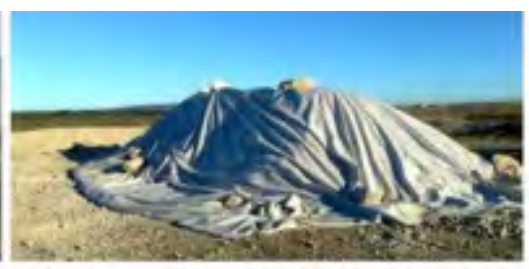

Rucupérotion et stockage ofu sable de surface (oraines) pour remise en ploce en fin de travoux

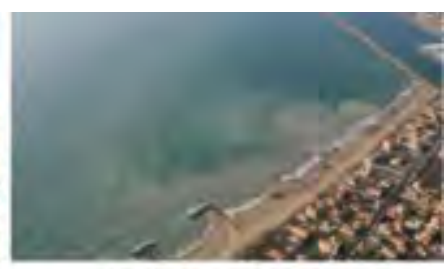

19/02/2015 - chantier en cours

Figure 8. Illustration des mesures de réduction des impacts.
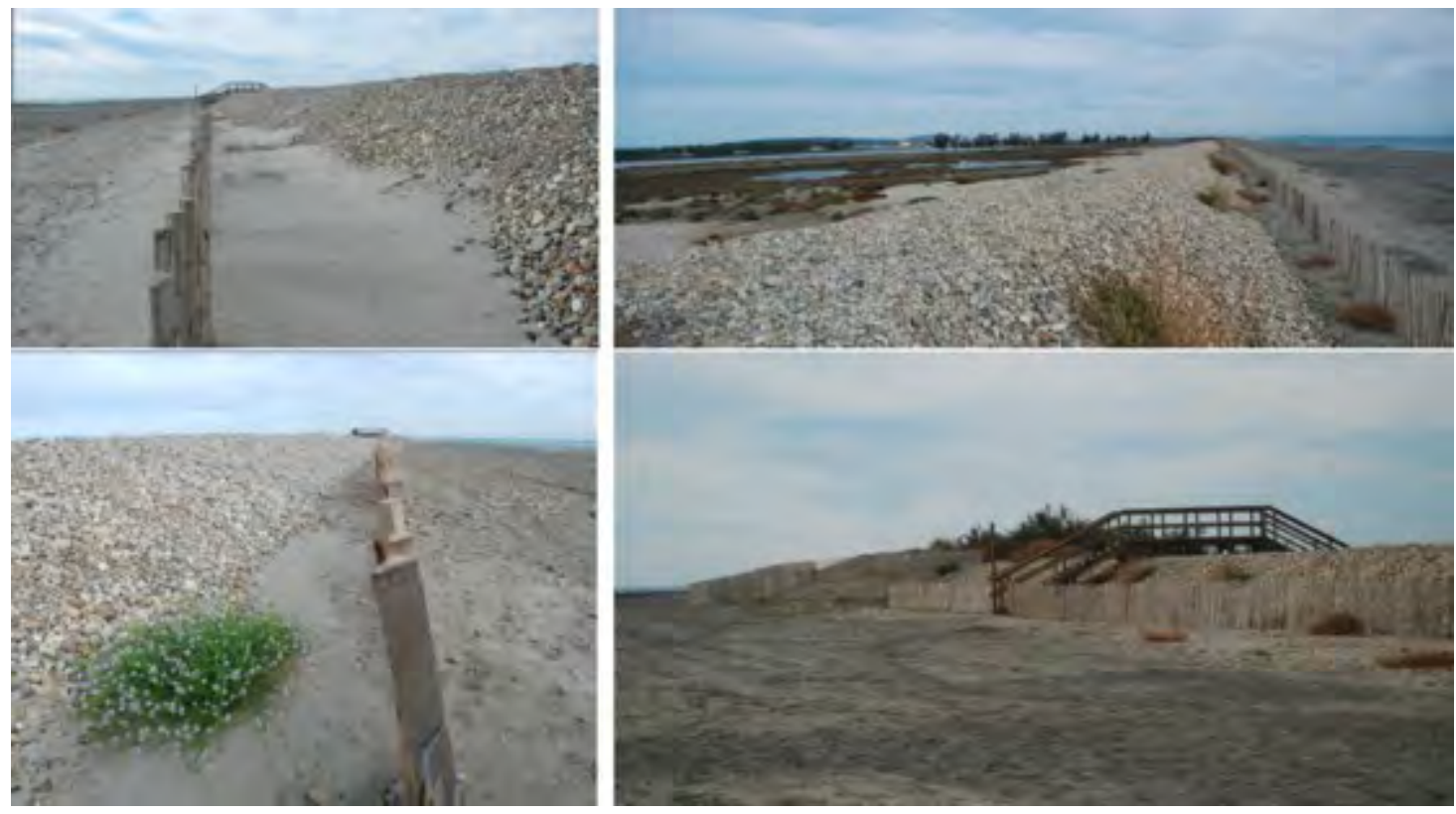

Figure 9. Vues de la plage une fois les travaux terminés.

\section{Remerciements}

Nous remercions vivement Thau AGGLO pour nous avoir permis la diffusion de ce document.

\section{Références bibliographiques}

CANDHIS -Centre d'Archivage National de Données de Houle In Situ- (site web). http://candhis.cetmef.developpement-durable.gouv.fr/. CETMEF.

CERC -Coastal Engineering Research Center- (1984). Shore Protection Manual, Vol. I \& II. Department of the Army, US Army Corps of Engineers. 